\title{
Atypical presentation of ileo-sigmoid knot - a rare case
}

\author{
Narasimha Reddy G ${ }^{1}$, Raja Sekhar G ${ }^{1}$, Md. Jawed Akther ${ }^{1 *}$ and Shameem Unnisa Shaik ${ }^{1}$ \\ Department of General Surgery, Mamata Medical College, Khammam, Telangana, India
}

\begin{abstract}
Ileosigmoid knot, also known as compound volvulus, is an unusual and a rare cause of intestinal obstruction. We are reporting a case of ileosigmoid knot in a 25 -yearold male, who presented with features of acute intestinal obstruction, peritonitis and hypovolemic shock. On immediate exploration after resuscitation, we found minimal haemorrhagic intraperitoneal fluid and gangrenous total colon, gangrenous distal ileal loop twisted around the base of the gangrenous sigmoid loop. Total colectomy with distal ilectomy $(50 \mathrm{cms})$, Hartmann's procedure with end ileostomy was done. After 3 months we performed ileorectal anastomosis.
\end{abstract}

\section{Introduction}

Ileosigmoid knot (ISK), also known as compound volvulus, is an unusual and rare cause of intestinal obstruction. In this condition, a loop of ileum encircles the loop of sigmoid colon \& then knots on itself. It generally occurs in areas with a high incidence of sigmoid volvulus [1]. ISK is an unusual entity in the West, but is comparatively common in certain African, Asian and Middle Eastern nations, known as 'volvulus belt'. The aetiology of ISK is controversial.ISK rapidly progress to gangrene of the ileum as well as of the sigmoid colon.

\section{Case report}

A 25-year-old male was brought to the emergency department with severe pain and distension of abdomen for 12 hours duration, which he developed in the later part of the night. Patient presented with vomitings and obstipation. On examination his pulse rate was 112/ min, having low volume, $\mathrm{BP}$ was $90 / 60 \mathrm{~mm} \mathrm{Hg}$ and respiratory rate was 30 cycles/min. Abdomen was distended without visible peristalsis. Abdominal examination revealed guarding and rebound tenderness without any audible bowel sounds. Patient was in hypovolemic shock, so resuscitated immediately. Investigations revealed leucocytosis (TLC-16,000), hypokalemia (Serum $\mathrm{K}^{+}-2.8 \mathrm{meq} / \mathrm{lit}$ ) and multiple air fluid levels along with dilated colonic loops in plain X-ray abdomen in erect position (Figure 1).

With a provisional diagnosis of acute large bowel obstruction and peritonitis, we proceeded for emergency exploratory laparotomy. On exploration, minimal hemorrhagic peritoneal fluid was noted. A gangrenous loop of ileum encircling base of loop of gangrenous sigmoid colon was found (Figure 2). Unknotting of ileosigmoid knot was successfully achieved and on further exploration, a kink was found in the ileum distal to the knot. Entire colon along with distal ileum except $10 \mathrm{~cm}$ of ileum adjacent to Ileo-caecal junction was gangrenous (Figure 3 and 4). Total colectomy with distal ilectomy $(50 \mathrm{cms})$ followed by Hartmann's procedure with end ileostomy in the right iliac fossa was done. Patient was allowed enteral nutrition on $3^{\text {rd }}$ postoperative day and was supplemented with multivitamins (vitamin $\mathrm{B}_{12}$ ). Postoperative period was uneventful and the patient was discharged on tenth post-operative day.

Patient was on regular follow-up and his general condition improved after 3 months when he was planned for relaparotomy and ileorectal anastomosis was done. Patient was allowed liquid diet on $5^{\text {th }}$ postoperative day and was gradually advanced to solid diet on $7^{\text {th }}$ postoperative day. Entire postoperative period was uneventful and was

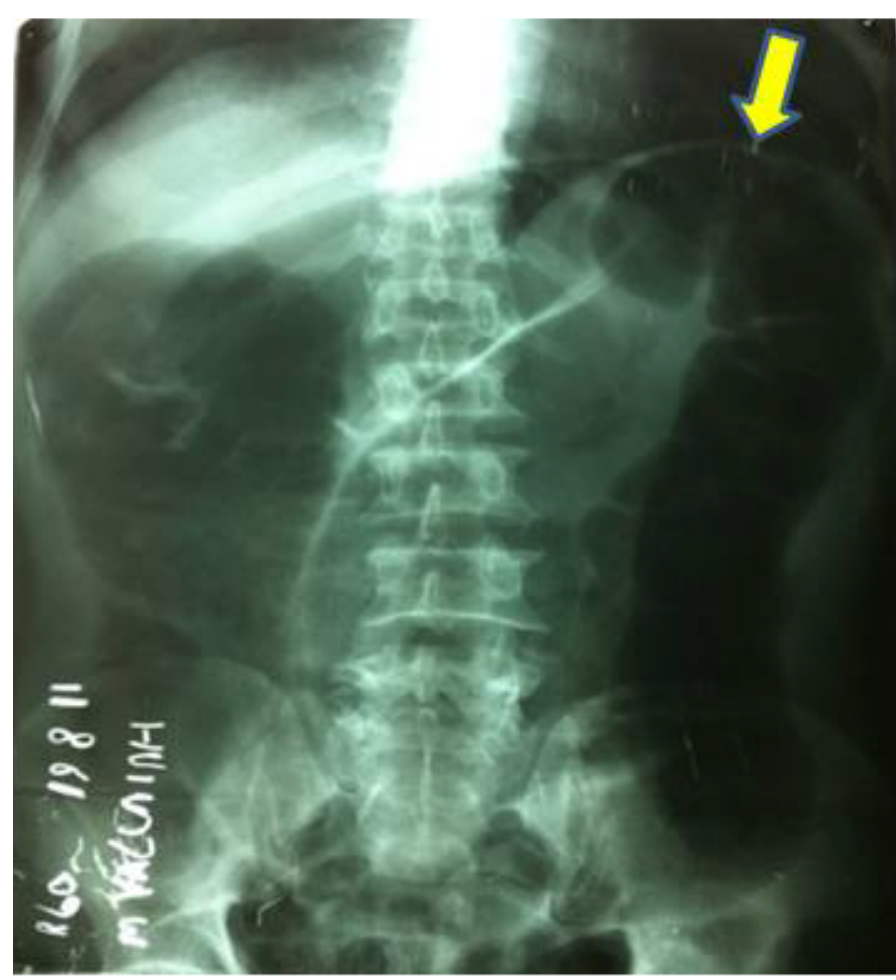

Figure 1. Xray showing dilated colon

Correspondence to: Md. Jawed Akther, Penna Apartment, Flat - 102, Mamata General Hospital Campus, Department of General Surgery, Mamata Medical College, Khammam, Telangana, India; E-mail: dr_mdjawedakther@yahoo.com

Key words: ileosigmoid knot, gangrenous total colon and distal ileum, hartmann's procedure with end ileostomy

Received: September 03, 2015; Accepted: September 28, 2015; Published: October 01, 2015 


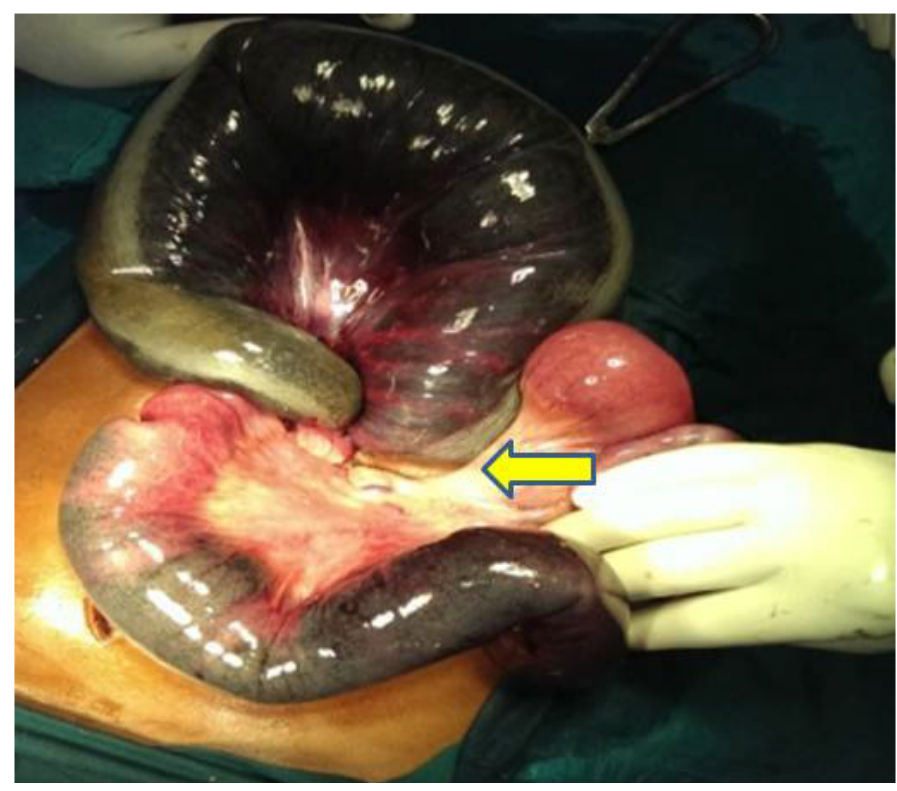

Figure 2. Showing ileosigmoid knot.

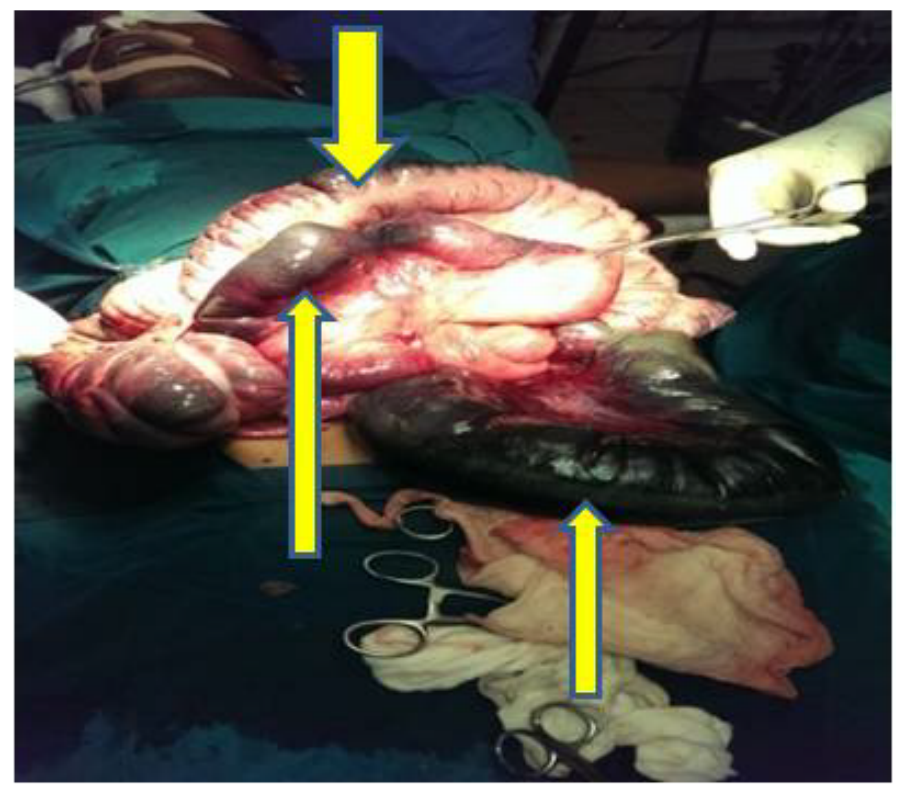

Figure 3. Showing gangrenous transvers colon, ileum, and sigmoid colon.

discharged on twelfth postoperative day. Patient is on regular followup for last 1 year and without any features suggestive of either bile salt or vitamin $\mathrm{B}_{12}$ malabsorption.

\section{Discussion}

Parker has been credited for describing the first patient of ileosigmoid knot in 1845. It more commonly affects men who are in fourth decade of life.Aetiology of ileosigmoid knot remains controversial [1]. This condition is common in east Africa, particularly among the young males of the Baganda tribe. Three factors are responsible for ileosigmoid knot; a long small bowel mesentery and a freely mobile small bowel, a long sigmoid colon on a narrow pedicle and finally, the ingestion of a high bulk diet in presence of an empty small bowel $[1,2,3]$. In our case all three factors were present. Ileosigmoid knot has been categorized into four types. In type I, ileum acts as active

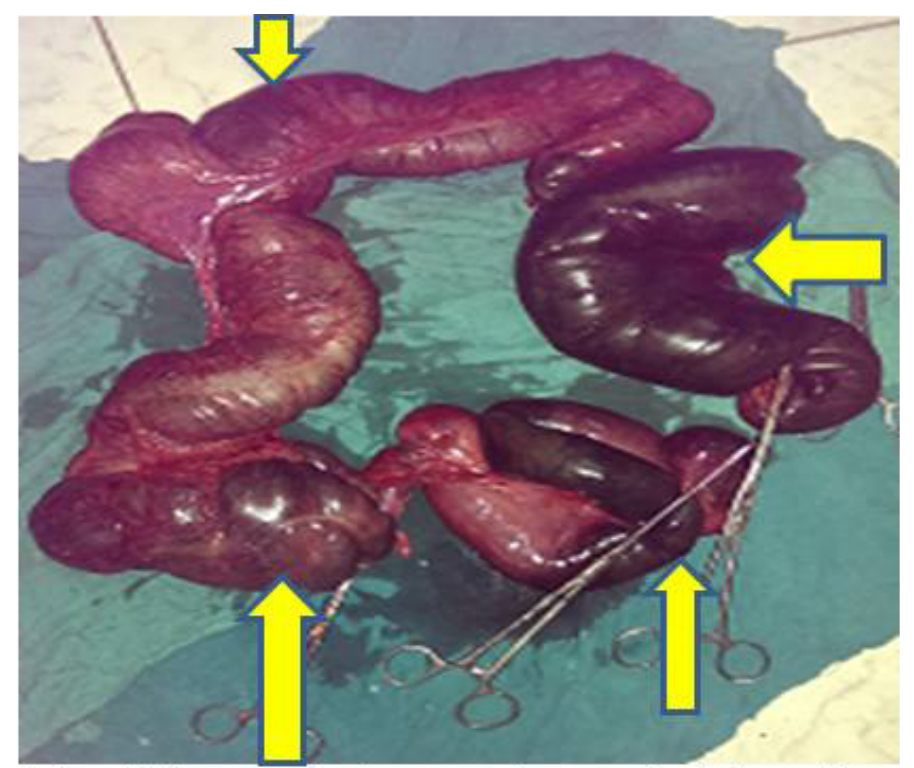

Figure 4. Resected specimen total colon and distal ileum.

component and wraps around the sigmoid colon. In type II, sigmoid colon is the active component wrapping itself around ileum. In both these types it is subdivided as type $\mathrm{A}$, when the direction of torsion is clockwise and type B when torsion is counter-clockwise. In type III, the Ileo-caecal segment acts as the active component, while in type IV (undetermined type) [1,4]. Our case was type I, A ileosigmoid knot. The predominant symptoms and signs of presentation include abdominal pain and tenderness (100\%), abdominal distension (94\% to $100 \%)$, nausea and vomiting ( $87 \%$ to $100 \%)$, rebound tenderness $(69 \%)$, and shock $(0 \%$ to $60 \%)[1,4,5]$. ISK can rapidly progress to gangrene of the ileum as well as of the sigmoid colon. Generalized peritonitis, sepsis, and dehydration are the principal complications. Radiographically, ISK is often mistaken for simple sigmoid volvulus. CT scan reveals the classical "whirl sign" of volvulus, created by the twisted mesentery and bowel, and the afferent and efferent limbs of the sigmoid colon have the appearance of a beak $[2,5]$.

The anatomical and pathological changes dictate the operative procedure. In $73.5 \%$ to $79.4 \%$ of the cases, gangrenous bowel was encountered, whereas in $20.6 \%$ to $26.5 \%$ both small and large bowels were assessed to be viable in surgery. In $52.9 \%$ to $60.3 \%$ cases, both the small intestine and sigmoid colon were gangrenous. Paradoxically, the incidence of bowel gangrene was $90.9 \%$ in those who presented within 24 hours of their symptoms. Among those who presented after 24 hours after their initial symptoms, bowel gangrene was seen in $57 \%$ $[2,4,6]$. In our case we found total colon along with distal ileum except $10 \mathrm{~cm}$ of ileum adjacent to Ileo-caecal junction were gangrenous. Closed loop obstruction caused distension of sigmoid loop, ileal loop \& the bowel between these two loops. Both strangulation and thrombosis of the vessels due to increased intramural pressure because of the closed-loop obstruction contributed to ischemia and gangrene of long segment of bowel except $10 \mathrm{~cm}$ of non-gangrenous ileum. This type of presentation we did not find to be reported in English literature even after doing through search, so a rare presentation. Various surgical procedures have been conducted in these patients depending upon the bowel viability [5]. 


\section{Conclusion}

Ileosigmoid knot as such is a very rare cause of intestinal obstruction and a high degree of suspicion is needed in cases mimicking sigmoid volvulus. Timely intervention is needed as it is a closed loop obstruction and that rapidly progresses to peritonitis and gangrene. It is uncommon to see cases presenting with total gangrenous colon and distal ileum which is an atypical presentation in our case and till now not reported in literature.

\section{References}

1. Shepherd JJ (1967) Ninety-two cases of ileosigmoid knotting in Uganda. Br J Surg 54 561-566. [Crossref]
2. Atamanalp SS, Oren D, Basoglu M, Yildirgan MI, Balik AA, et al. (2004) Ileosigmoid knotting: outcome in 63 patients. Dis Colon Rectum 47: 906-910.

3. Akgun Y (1997) Management of ileosigmoid knotting. Br J Surg 84: 672-673. [Crossref]

4. Alver O, Oren D, Tireli M, Kayabaşi B, Akdemir D (1993) Ileosigmoid knotting in Turkey. Review of 68 cases. Dis Colon Rectum 36: 1139-1147. [Crossref]

5. Norman O. Machado (2009) Ileosigmoid knot: a case report and literature review of 280 cases. Ann Saudi Med 29: 402-406. [Crossref]

6. Atamanalp SS, Oren D, Yildirgan MI, Başoğlu M, Aydinli B, et al. (2007) Ileosigmoidal knotting in children: a review of 9 cases. World J Surg 31: 31-35. [Crossref]

Copyright: $(2015$ Narasimha Reddy G. This is an open-access article distributed under the terms of the Creative Commons Attribution License, which permits unrestricted use, distribution, and reproduction in any medium, provided the original author and source are credited. 